\section{Compound heterozygous SCN5A gene mutations in asymptomatic Brugada syndrome child}

\author{
Elena Sommariva, ${ }^{1}$ Matteo Vatta, ${ }^{2}$ \\ Yutao Xi, ${ }^{3}$ Simone Sala, ${ }^{4}$ Tomohiko $\mathrm{Ai},{ }^{5}$ \\ Jie Cheng, ${ }^{3}$ Carlo Pappone, ${ }^{6}$ \\ Maurizio Ferrari, ${ }^{1,7,8}$ Sara Benedetti ${ }^{7}$ \\ ${ }^{1}$ Genomic Unit for the Diagnosis of \\ Human Pathologies, Centre for Genomics, \\ Bioinformatics and Biostatistics, San \\ Raffaele Scientific Institute, Milano, Italy; \\ 2Department of Pediatrics, Section of \\ Cardiology, Baylor College of Medicine, \\ Houston, TX, USA; ${ }^{3}$ Electrophysiology \\ Research Laboratory, Texas Heart \\ Institute/St. Luke's Episcopal Hospital, \\ Houston, TX, USA; ${ }^{4}$ Unit of \\ Arrhythmology, Cardiotoracovascular \\ Department, San Raffaele Scientific \\ Institute, Milano, Italy; ${ }^{5}$ Krannert \\ Institute of Cardiology, Indiana \\ University, Indianapolis, IN, USA; \\ ${ }^{6}$ Department of Arrhythmology, Maria \\ Cecilia Hospital, Cotignola, Italy; \\ ${ }^{7}$ Laboratory of Clinical Molecular Biology, \\ Diagnostica e Ricerca San Raffaele S.p.A., \\ Milano, Italy; ${ }^{8}$ Vita-Salute San Raffaele \\ University, Milano, Italy
}

\section{Abstract}

Loss-of-function mutations in the SCN5A gene, encoding the cardiac Nav1.5 sodium channel, have been previously associated with Brugada syndrome (BrS). Despite the low prevalence of the disease, we identified a patient carrying two SCN5A mutations. We aimed at establishing a correlation between genotype, clinical phenotype and in vitro sodium current. A 3-year-old boy presented with right bundle branch block and ST-segment elevation. Genetic analysis and electrophysiology studies in transfected HEK293 cells were performed to identify possibly disease-causing variants and assess their effect on sodium channel function. Two SCN5A variants were identified: a new frameshift deletion causing premature truncation of the putative protein (c.3258_3261del4) and a missense substitution (p.F1293S). In vitro studies revealed that the truncated mutant did not produce functional channels and decreased total sodium current when co-expressed with p.F1293S channels compared to p.F1293S alone. In addition, p.F1293S channels presented with a steep slope of steady-state activation voltagedependency, which was shifted towards more positive potentials by the co-expression with the truncated channel. p.F1293S channels also showed shift towards more positive potentials of the steady-state inactivation both alone and co-expressed with the deletion mutant.

Our data identified a severe reduction of sodium channel current associated with two distinct SCN5A changes. However, all mutation carriers were asymptomatic and BrS electrocardiogram was observed only transiently in the compound heterozygous subject. These observations underline the difficulty of genotype/phenotype correlations in BrS patients and support the idea of a polygenic disorder, where different mutations and variants can contribute to the clinical phenotype.

\section{Introduction}

Brugada syndrome (BrS) is an autosomal dominant arrhythmogenic syndrome that can be associated with ventricular fibrillation and sudden cardiac death in patients with structurally normal heart. BrS was first described in 1992 as a distinct clinical and electrocardiographic entity, characterized by ST-segment elevation in the right precordial leads (V1-V3) and right bundle branch block at surface electrocardiogram (ECG). ${ }^{1}$ However, the BrS ECG pattern is often concealed and can be unmasked using sodium channel blockers. To date, the implantable cardioverter defibrillator (ICD) represents the only viable option for symptomatic patients to prevent the occurrence of sudden death from malignant arrhythmias. ${ }^{2}$ However, new criteria for prognostic risk stratification of these patients are needed, especially for asymptomatic subjects. ${ }^{3}$ Despite the extraordinary progresses in characterizing $\mathrm{BrS}$, still little is known about its natural history in the pediatric population. It has been however reported that BrS children display diagnostic and prognostic features similar to adult patients and may be as well subjected to sudden death. ${ }^{4}$

In 1998 Chen associated $\mathrm{BrS}$ with mutations in the $S C N 5 A$ gene, ${ }^{5}$ encoding the $\alpha$-subunit of the voltage-gated cardiac sodium channel Nav 1.5. ${ }^{6}$ However, only $20-25 \%$ of clinically diagnosed BrS patients carry a SCN5A mutation. ${ }^{7-9}$ At present, over three hundred SCN5A mutations have been associated with $\mathrm{BrS}$ (http://www.fsm.it/cardmoc/), which generally reduce the magnitude of the cardiac sodium current in functional studies by a variety of mechanisms. ${ }^{10-12}$ However, the emerging concept of arrhythmia genomics supports the idea of a complex disorder, where the co-segregation of different mutations and common genetic variants can contribute to the clinical phenotype. ${ }^{13}$

Here we report a three years-old child diag-
Correspondence: Sara Benedetti, Laboratory of Clinical Molecular Biology, Diagnostica e Ricerca San Raffaele, via Olgettina 60, 20132 Milano, Italy. Tel: $+39-02-26432534$ - Fax: $+39-02-2643$ 4351. E-mail benedetti.sara@hsr.it

Key words: Brugada syndrome, arrhythmia, genetics, double mutant, sodium channel.

Acknowledgements: we are indebted to $\mathrm{Dr}$ Francesco M. Sacco for help in clinical assessment, Miss Erica W. Taylor for generation ofSCN5A mutated plasmids and Dr.Chiara Di Resta for critically reading the manuscript and help with editing.

Contributions: ES, SB, concept/design, genetic data collection, analysis and interpretation, article drafting, critical revision and approval; MV, electrophysiological data collection, analysis and interpretation, article critical revision and approval; YX, TA, JC, electrophysiological data collection and analysis, article drafting and approval; SS, clinical data collection, article drafting and approval; CP, MF, concept/design, clini$\mathrm{cal} /$ genetic data interpretation, funding, article critical revision and approval.

Conflict of interests: the authors declare no potential conflict of interests.

Funding: this work was supported by grants from Medtronic Italy (to M.F.) and the Italian Istituto Superiore di Sanità (ISS-526D/55 to C.P.).

Received for publication: 1 June 2012.

Revision received: 30 July 2012 .

Accepted for publication: 3 August 2012

This work is licensed under a Creative Commons Attribution NonCommercial 3.0 License (CC BYNC 3.0).

(OCopyright E. Sommariva et al., 2012

Licensee PAGEPress, Italy

Cardiogenetics 2012; 2:e11

doi:10.4081/cardiogenetics.2012.e11

nosed with BrS and carrying two different SCN5A mutations inherited from his asymptomatic parents. In addition, we describe the in vitro electrophysiological characterization of the two variants. The difficulty in correlating genotype with phenotype underlines the importance of widening the genetic screening of BrS patients to identify modifier variants.

\section{Materials and Methods}

\section{Clinical phenotype}

A 3-year-old boy of Caucasian ethnical origin was referred to our center for routine control related to occasionally detected congenital heart murmur. Echocardiography examination 
revealed oval foramen perviety without hemodynamic impairment, but was otherwise negative, with a conserved ejection fraction (75\%). Chest X-ray was negative. ECG at admission showed sinus rhythm with focal right bundle branch block (Figure 1A). However, a second ECG showed a coved ECG ST elevation pattern ( $>2$ $\mathrm{mV}$ ) in $\mathrm{V} 1$ and $\mathrm{V} 2$ leads with $\mathrm{T}$ wave inversion in leads V1, V2 and V3, unmasking a typical type I BrS ECG pattern according to criteria indicated in the consensus report ${ }^{14}$ (Figure 1B). The duration of PR interval was 180-200 $\mathrm{ms}$, with $\mathrm{QRS}=100 \mathrm{~ms}$ in V4. During $24 \mathrm{~h}$ Holter-ECG recording (mean frequency 90 bpm, minimum 82 bpm, max 139 bpm) a selfterminating asymptomatic episode of polymorphic ventricular tachycardia (130 bpm) was recorded. Also in consideration of the very young age, the patient was not subjected to ICD implantation and a close follow-up was proposed with serial ECG and $24 \mathrm{~h}$ Holter monitoring and prompt antipyretic treatment during fever and before immunizations. Proband parents were completely asymptomatic with normal echocardiography. However, both ECGs showed right ventricular conduction delay (Figure 1C and D). Unfortunately, they refused to undergo further clinical examination including flecainide testing. ECG parameters of all three family members are showed in Table 1.

\section{Genetic analysis}

Written informed consent was obtained before genetic analysis. The investigation conformed to the principles outlined in the Declaration of Helsinki. Genomic DNA was extracted from peripheral blood using the EZ1 Bio-Robot extractor (Qiagen, Venlo, the Netherlands). Molecular analysis was conducted on SCN5A coding sequence, including flanking intronic regions, by polymerase chain reaction amplification. Mutation screening was performed using Denaturing High Performance Liquid Chromatography (Transgenomic, Omaha, NE, USA) and automated direct sequencing (ABI 3730, Applied Biosystems Inc., Foster City, CA, USA). Primer sequences are available on request. SCN5A NCBI reference sequences are NM_198056 (nucleotides are numbered starting from the ATG) and NP_932173.

\section{Constructs preparation}

The c.3258 3261del4 and c.3878T >C (p.F1293S) SCN5A mutants were prepared by site-directed mutagenesis using Quick Change ${ }^{\circledR}$ Site-Directed Mutagenesis Kit (Stratagene, La Jolla, CA, USA) and plasmid pcDNA3.1-SCN5A as template, which contains the hH1 clone as previously described..$^{15}$ $S C N 5 A$ cDNA was amplified using $\mathrm{Pfu}^{+}$DNA polymerase (Stratagene) and the following oligonucleotides: 5' -GCCTGTGTCCGGTGGCC-
CGCCCCTCCGGATTCCAG-3' (sense) and 5'CTGGAATCCGGAGGGGCGGGCCACCGGACACAGGC-3' (antisense) for c.3258_3261 del4, and 5 '-GCCAACACCCTGGGCTCTGCCGAGATGGGCCCCA-3' and 5'- TGGGGCCCATCTCG GCAGAGCCCAGGGTGTTGGC-3' for p.F1293S. The mutated c.3258_3261del4 and p.F1293S clones were sequenced to ensure the presence of the mutations and the absence of spurious variations.

\section{Transient expression of wild-type and mutant channels}

Transient transfection of wild-type (WT) and mutant SCN5A cDNA was performed as previously described. ${ }^{16}$ To characterize WT and mutant SCN5A separately, HEK293 (human embryonic kidney) cells were transfected with $5 \mu \mathrm{g}$ of pcDNA3.1 containing WT SCN5A cDNA, p.F1293S or c.3258_3261del4 using Lipofectamine 2000 transfection reagent (Invitrogen, Carlsbad, CA, USA). To mimic the heterozygote situation, $2.5 \mu \mathrm{g}$ of p.F1293S and $2.5 \mu \mathrm{g}$ of c.3258_3261del4 were co-transfected.

\section{Patch-clamp experiments and data analysis}

Patch-clamp experiments were performed according to the previously described methods $\cdot{ }^{16}$ Whole-cell recordings were carried out at
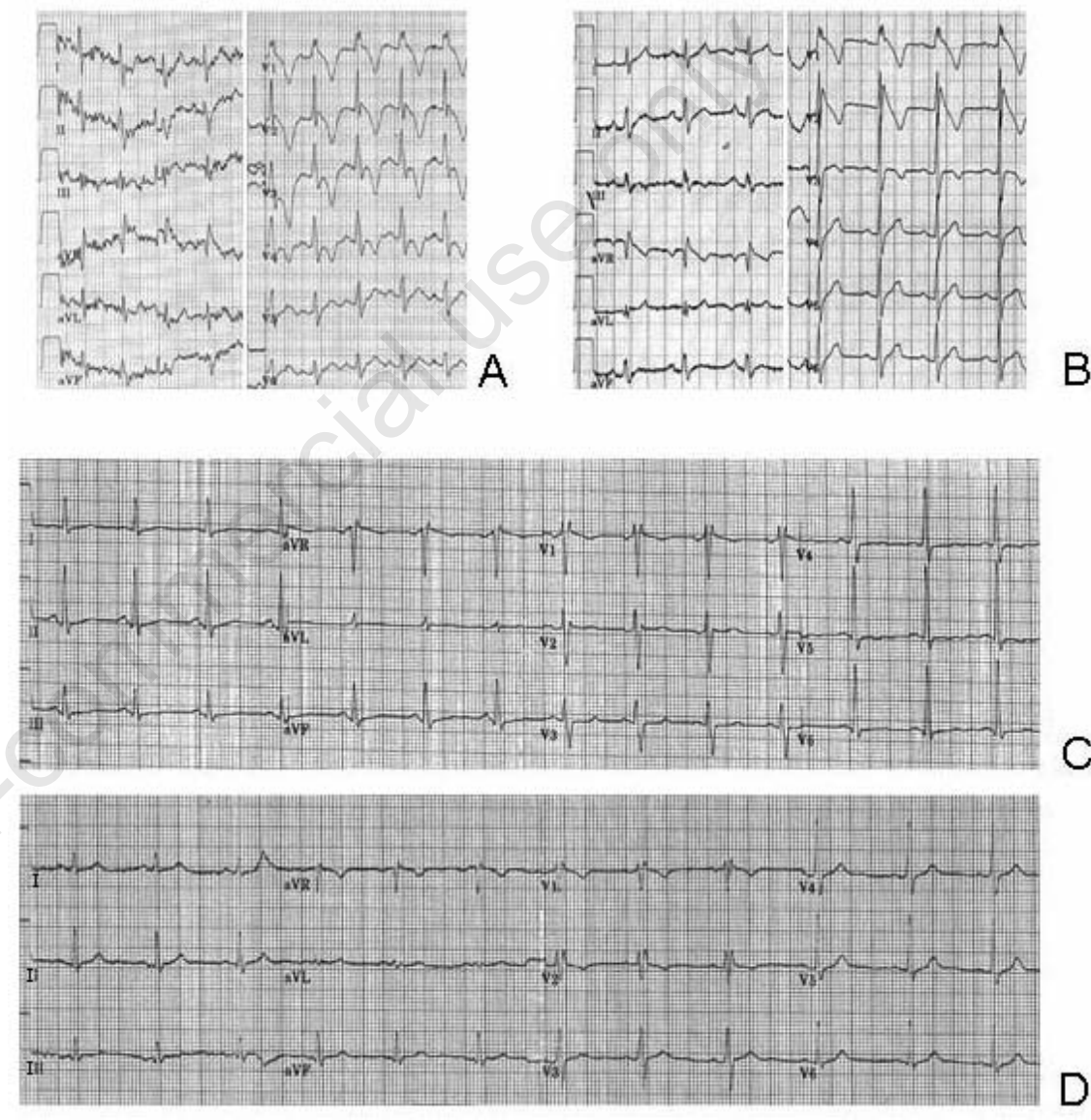

Figure 1. Twelve-lead surface electrocardiogram (ECG). (A) Proband, first ECG showing sinus rhythm with focal right bundle branch block; (B) Proband, second ECG revealing sinus bradycardia with typical type I Brugada syndrome $(\mathrm{BrS})$ pattern in V1 and V2 . In both panels the electrodes were placed in the same position. (C) Mother ECG and (D) Father ECG, showing sinus rhythm and no type I BrS pattern.

Table 1. Electrocardiogram intervals duration (ms). In parenthesis reference values.

\begin{tabular}{lccc} 
& PR & QRS & OIc \\
Proband & $180-200(100-150)$ & $100(90-110)$ & $395(<440)$ \\
Mother & $160(120-200)$ & $110(70-110)$ & $430(<460)$ \\
\hline Father & $150(120-200)$ & $108(70-110)$ & $440(<450)$ \\
\hline
\end{tabular}


$22^{\circ} \mathrm{C}$. Step-pulse voltage protocols were generated with Axopatch 200B using Pclamp 9 software (Molecular Devices, Sunnyvale, CA, USA). The pipette solution contained $10 \mathrm{NaF}$, $110 \mathrm{CsF}, 20 \mathrm{CsCl}, 10 \mathrm{EGTA}$, and $10 \mathrm{HEPES}$ (in $\mathrm{mM}$, pH 7.35 with $\mathrm{CsOH}$ ). Bath solution contained $145 \mathrm{NaCl}, 4 \mathrm{KCl}, 1 \mathrm{MgCl}_{2}, 1.8 \mathrm{CaCl}_{2}, 10$ HEPES, and 10 glucose (in $\mathrm{mM}, \mathrm{pH}=7.35$ with $\mathrm{NaOH})$.

Conductance $\mathrm{G}(\mathrm{V})$ was calculated by the equation:

$$
\mathrm{G}(\mathrm{V})=\mathrm{I} /\left(\mathrm{V}_{\mathrm{m}}-\mathrm{E}_{\text {rev }}\right)
$$

where I is the peak currents, $\mathrm{E}_{\mathrm{rev}}$ is the measured reversal potential, and $\mathrm{V}_{\mathrm{m}}$ is the membrane potential.

The normalized peak conductance was plotted against membrane potentials. Steady-state inactivation was estimated by a pre-pulse protocol (500 ms from a holding potential of -140 $\mathrm{mV}$; shown in the inset). The normalized peak currents were plotted as a function of membrane potentials. Steady-state activation and inactivation were fitted with the Boltzmann equation:

$$
\mathrm{y}=\left[1+\exp \left(\left(V_{h}-V_{\mathrm{m}}\right) / k\right)\right]-1
$$

where y represents variables; $V_{h}$, midpoint; $k$, slope factor; $V_{m}$, membrane potential.

The biophysical characteristics of sodium channels were analyzed using Clampfit (Axon Instruments, Sunnyvale, CA, USA) and Igor software (Wavemetrics, Lake Oswego, OR, USA). Data were presented as mean \pm SD. Comparisons among data were made using one-way ANOVA followed by Tukey test with $\mathrm{P}<0.05$ considered as statistically significant.

\section{Results}

\section{Genetic analysis}

SCN5A molecular analysis identified in the proband two variants compatible with $\mathrm{BrS}$ (Figure 2): the first was a novel heterozygous four nucleotides deletion in exon 18 (c.3258_3261del4, from now on named Del4), leading to frameshift and generation of a premature stop codon in the predicted protein after 56 amino acids, within the cytoplasmic linker between domains DII and DIII (p.E1087PfsX57). The second was a c.3878T >C heterozygous nucleotide substitution in exon 22 , leading to the aminoacidic change p.F1293S (from now named FS). This variant, located in the extracellular linker between S2$\mathrm{S} 3$ in domain DIII, had been previously reported both in a BrS patient ${ }^{17}$ and in apparently healthy individuals with a frequency of 1/295 among Caucasians from the US, ${ }^{18}$ therefore its pathogenicity was unclear. The analysis by the computer-based algorithms PolyPhen-2 (http://genetics.bwh.harvard.edu/pph2/) and SIFT (http://sift.jcvi.org/) suggested that the FS substitution might be tolerated.

We subsequently performed a targeted mutation analysis on the proband's parents to determine the configuration of the c.3258_3261del4 and c.3878T >C alleles, due to the different putative functional effect on the sodium channel subunits. Indeed, molecular analysis revealed that the two changes identified in the index case were in a trans configuration, with the FS missense mutation inherited from the father and the Del4 frameshift deletion from the mother, consistently with the proband being a compound heterozygote (Figure 2).

\section{In vitro electrophysiology}

In order to determine how the two variants could affect cardiac sodium currents, in vitro electrophysiology studies were performed. We generated two plasmids carrying Del4 or FS variants by site-directed mutagenesis and the biophysical characteristics of the mutants were studied and compared to WT channels using whole-cell patch-clamp methods.

Figure 3A shows representative macroscopic current traces obtained from HEK293 cells transiently transfected with SCN5A WT, FS, Del4 or co-transfection of FS+Del4 to mimic the proband's compound heterozygous condition. Figure 3B shows their current-voltage relationships, suggesting that current density was significantly attenuated in the cells trans-

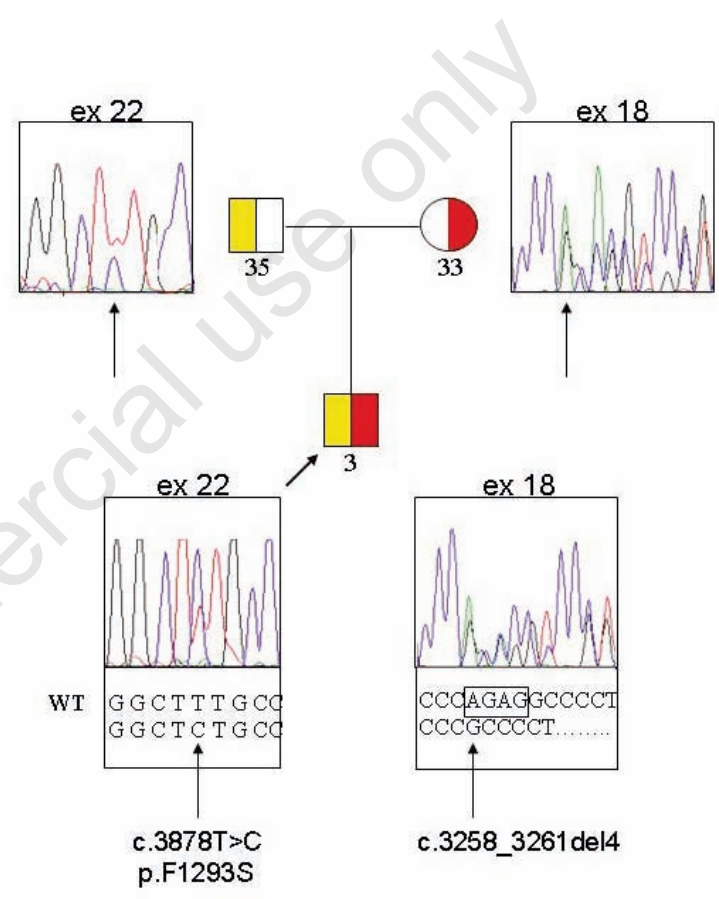

Figure 2. Molecular results of the genetic analysis on $S C N 5 A$ exon 18 and 22. Father's sequence shows the $T>C$ base heterozygous substitution in position c.3878 in exon 22 (p.F1293S). Mother's sequence presents the heterozygous four bases deletion (c.3258_3261del4) in exon 18. The proband's sequence shows both mutations. Yellow represents the point mutation in exon 22, red the deletion in exon 18.

Table 2. Kinetic parameters for wild-type, p.F1293S, Del4 and p.F1293S+Del4 sodium channels.

\begin{tabular}{lcccc} 
& WT & FS & Del4 & FS+Del4 \\
Current density $(\mathrm{pA} / \mathrm{pF})$ & $(10)$ & $(10)$ & $(10)$ & $(10)$ \\
& $-259.7 \pm 7.0$ & $-150.7 \pm 5.9^{*}$ & $-1.26 \pm 0.22^{* *}$ & $-64.1 \pm 2.5^{*}$ \\
Activation & $(10)$ & $(10)$ & & $(10)$ \\
$\mathrm{V}_{\mathrm{h}}(\mathrm{mV})$ & $-40.6 \pm 0.6$ & $-41.0 \pm 0.3$ & $\mathrm{NA}$ & $-31.6 \pm 0.3^{* * \circ}$ \\
$\mathrm{k}$ & $7.6 \pm 0.5$ & $5.1 \pm 0.2^{*}$ & $\mathrm{NA}$ & $7.7 \pm 0.3$ \\
\hline Fast-inactivation & $(10)$ & $(8)$ & & $(9)$ \\
$\mathrm{V}_{\mathrm{h}}(\mathrm{mV})$ & $-91.4 \pm 0.1$ & $-84.3 \pm 0.2^{* *}$ & $\mathrm{NA}$ & $-84.4 \pm 0.6^{* *}$ \\
$\mathrm{k}$ & $7.8 \pm 0.1$ & $7.1 \pm 0.2$ & $\mathrm{NA}$ & $6.6 \pm 0.4$ \\
\hline
\end{tabular}

Data were represented as mean \pm SD. The numbers in parenthesis represent number of patches. WT, wild-type; FS; p.F1293S; $k$, slope factor; $V_{h}$, midpoint; NA, not applicable; ${ }^{*} \mathrm{P}<0.05$ and ${ }^{* *} \mathrm{P}<0.01$ us $\mathrm{SCN} 5 \mathrm{~A}-\mathrm{WT} ;{ }^{\circ} \mathrm{P}<0.01$ us FS. 
fected with FS. On the other hand, no significant current was obtained from cells transfected with Del4 alone (Table 2). Accordingly, coexpression of FS and Del4 showed further decreased current compared to FS alone (from $58 \%$ to $24 \%$ compared to WT).

The normalized conductance of WT, FS and co-expression of FS+Del4 was then plotted against the membrane potentials (Figure 3C). Steady-state inactivation was examined by a pre-pulse protocol and normalized currents were plotted as a function of membrane potentials. Data were fitted with the Boltzman's equation (see Materials and Methods section, for data analysis): co-expression of FS+Del4 was found to shift the voltage-dependency of steady activation towards more positive potentials by about $10 \mathrm{mV}(\mathrm{P}<0.01$ vs WT or FS), but FS alone had no significant effect $(\mathrm{P}=0.12$ vs WT) (Table 2). Furthermore, FS alone and FS+Del4 were found to shift towards more positive potentials the voltage-dependency of the steady-state inactivation by about $7 \mathrm{mV}$ ( $\mathrm{P}<0.01$ vs WT). In addition, FS rendered the conduction-voltage relation steeper than the WT (slope factor, $5.1 \pm 0.2$ vs $7.6 \pm 0.5, \mathrm{P}<0.05$ ) (Table 2). There was no further difference in steady state activation and inactivation. A magnified illustration in the inset indicates the window currents, reflecting the current densities of WT and mutant channels: the coexpression of Del4 shifted the activation curve of FS towards more positive values, which might contribute to the further decreased current density by Del4.

\section{Discussion}

Although $\mathrm{BrS}$ is considered a rare disease, more than 300 mutations in the SCN5A gene have been identified to date, generally transmitted in a dominant fashion. ${ }^{7}$ However, the incomplete penetrance and variable expressivity in BrS suggests that compound heterozygosis in each of the BrS-causing genes or digenic inheritance with double heterozygote BrS subjects may be determine and modulate the clinical phenotype.

The present study reports a BrS patient carrying two heterozygous SCN5A gene variants in a trans configuration. The first mutation (c.3258_3261del4) is a novel frameshift deletion in exon 18, creating a premature stop codon in the cytoplasmic linker between domains DII and DIII, which is compatible with loss of function of the sodium channel. The second change (p.F1293S) remains controversial, because it had been previously identified in a BrS patient ${ }^{17}$ and in apparently healthy individuals. ${ }^{18}$ Although computer-based algorithms PolyPhen-2 and SIFT indicated this substitution may be tolerated, its localization in the extracellular linker between S2-S3 in domain DIII, close to the voltage sensor segment, and the aminoacid conservation among different species suggests a possible pathogenic role.

Clinically, the three year-old proband, who is compound heterozygote for the aforementioned SCN5A changes, presented with heart murmur and displayed transient BrS type I ECG pattern with right bundle branch block and inverted $\mathrm{T}$ waves. The patient did not show other symptoms, although Holter monitoring recorded an isolated episode of self-terminating ventricular tachycardia. The parents, each carrier of either change, were completely asymptomatic and failed to display a BrS ECG phenotype. The mother carried the most severe mutation (Del4), while the father harbored the FS substitution, leading to a lesser decrease of sodium current. Gender is known to play a role in the variable expressivity of BrS. In fact, despite equal genetic transmission of the mutation, the clinical phenotype is 8 to 10 times more prevalent in males than in females..$^{19}$ We may speculate that the mother, carrying the frameshift deletion mutation, did not present with symptoms because she was protected by the female sex and the father carried a relatively mild mutation.
The effects of these variants were studied by heterologous expression of the mutated channels in HEK293 cells. Both mutations significantly reduced the amplitude of sodium current compared with WT and were thus expected to generate the substrate for the BrS phenotype. Accordingly, loss of $\mathrm{Na}^{+}$current by $\mathrm{BrS}$ mutations has been linked to three primary mechanisms: (i) truncation of the $\mathrm{Na}^{+}$channel $\alpha$-subunit, which yields a non-functional channel; ${ }^{20}$ (ii) alteration in channel gating, such as changes in activation, inactivation, or reactivation kinetics ${ }^{21}$ and (iii) altered trafficking of the channels through the endoplasmic reticulum/Golgi complex to the plasma membrane. ${ }^{22}$ A common denominator of these mutations is a reduced cardiac $\mathrm{Na}^{+}$current leaving the transient outward $\mathrm{K}^{+}$current $\left(\mathrm{I}_{\mathrm{t} 0}\right)$ unopposed in phase 1 and loss of the action potential dome in the right ventricular epicardium. In the present study, Del4 mutant sodium channels exhibit complete loss of function, while FS showed reduced current and a shift towards more positive potentials of the voltage-dependency of the steady-state inactivation, which might also cause loss of channel function. Coexpression of FS+Del4 mutants halved current compared to FS alone (58\% to $24 \%$ with respect

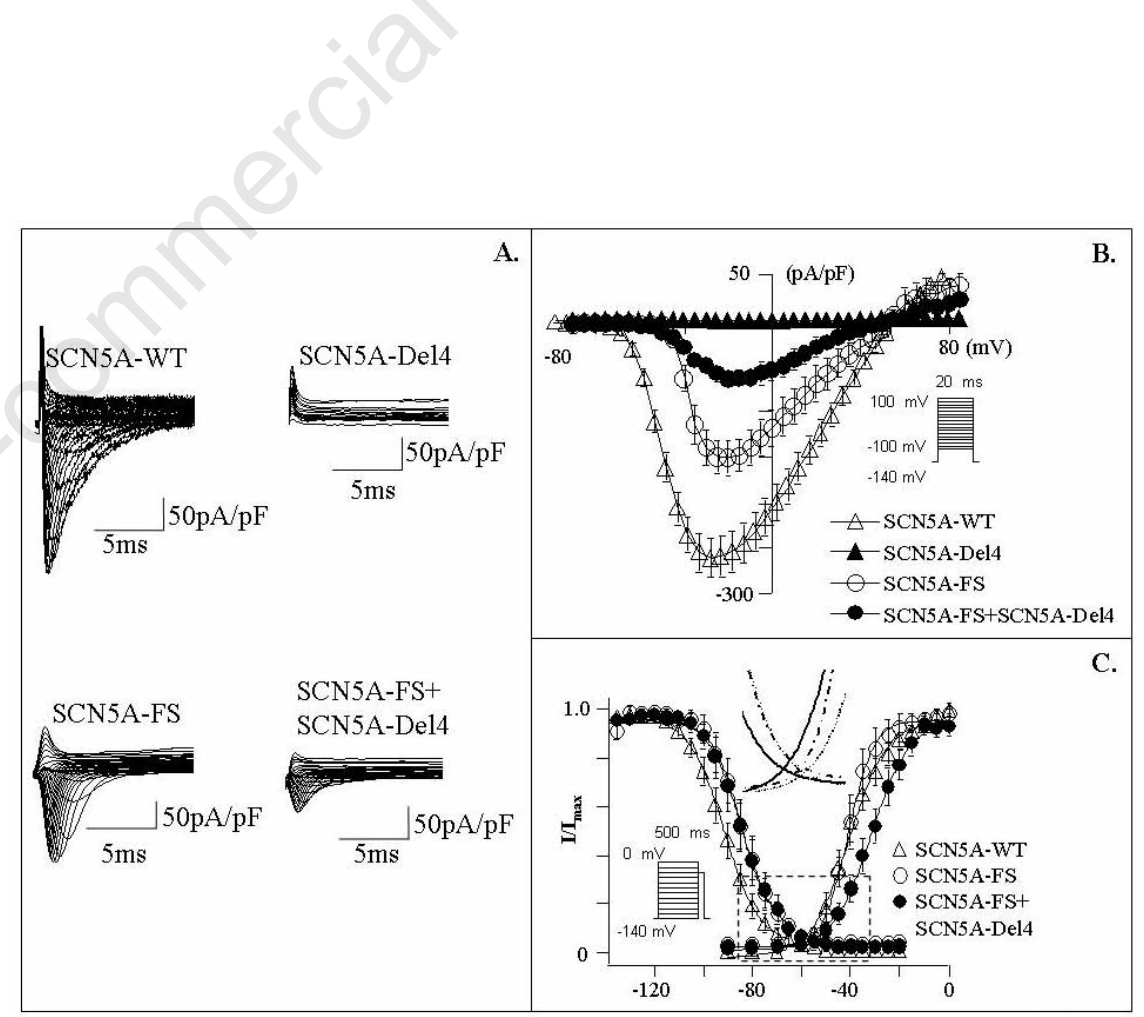

Figure 3. Patch clamp current traces and current-voltage relationships of p.F1293S (FS), Del4, and FS+Del4 mutations in HEK293 cells. (A) Superimposed whole-cell current traces induced by a step-pulse protocol from a holding potential of $-140 \mathrm{mV}$. (B) Currentvoltage relationship of peak current densities. (C) Voltage-dependence of peak conductance and steady-state fast inactivation. The inset indicates the magnified illustration of the window currents within the dash square. The solid line represents wild type; the dotdash line represents FS mutant and the dot line represents co-expression of FS and with Del 4 mutants. The data were represented as mean \pm S.E.M. 
to WT). This observation, which can be attributed to differences in the amount of FS DNA transfected (2.5 $\mu \mathrm{g}$ in co-transfection vs $5 \mu \mathrm{g}$ in single transfection), may mimic the situation in the proband, harboring only a partially functional allele and a null one. In the case of the father, heterozygous carrier of FS mutation, probably the current will be higher than the $58 \%$ recorded in in vitro experiments, because of the presence of the WT allele. However, we chose to measure current of FS channels alone, in order to investigate current reduction caused by the mutation without interference of the WT SCN5A.

Only a brief and occasional episode of atypical ventricular tachycardia was documented in the proband during Holter monitoring and he has been asymptomatic ever since in a two years follow up. Indeed, the identification of new SCN5A changes in asymptomatic cases can lead to controversial interpretation, because of the difficulty in establishing correlations with the phenotype and the risk of malignant arrhythmic events. Asymptomatic individuals with a BrS ECG pattern generally have a better prognosis than their symptomatic counterparts, but this may not be always the case. Asymptomatic patients carrying functionally proven SCN5A mutations with loss-offunction properties should therefore be followed carefully to avoid potentially lethal events. In this case, since the proband missed any WT copy of the gene, and hence any fully functional sodium channel, a severe phenotype would have been expected. Of course, given the young age of the proband, a longer follow up will be necessary to verify these findings. However, the clinical consequence of SCN5A mutations may also be greatly influenced by unknown environmental factors and genetic modifiers.

The identification of double SCN5A mutants raises the possibility that many patients with a genetic SCN5A alteration may carry additional variants in the same or other genes, further complicating the genetic picture. Indeed, in spite of the low prevalence of SCN5A alterations, double mutants have been described in few cases to date, both in the same ${ }^{23,24}$ and in different alleles. ${ }^{25-27}$ Generally, in these cases the two variants consisted of a mutation associated with a rare polymorphism, which may have a modulator effect. However, another study reported an infant carrying two SCN5A mutations in trans, which co-expressed in vitro elicited a significant increase in late sodium current not present in either mutant alone, suggesting interaction between the mutant proteins. ${ }^{28}$ In addition, combination of different loss-of-function mutations in depolarizing channel genes has been described in infants with BrS-like phenotype..$^{29}$ Only a wider genetic screening with high throughput technologies will therefore allow a better under- standing of genotype/phenotype correlations and pathogenetic mechanisms, which in turn will improve patient clinical management.

\section{Conclusions}

In summary, we found two heterozygous SCN5A gene mutations impairing the function of sodium channels in a BrS asymptomatic child and his parents. Genetic analysis confirmed therefore the diagnosis of BrS, ensuring him a proper follow-up, and prompted the identification of two asymptomatic individuals who might be at risk of potentially life-threatening arrhythmias. Although patch-clamp analysis revealed that each mutation produced a severe reduction of $\mathrm{Na}^{+}$current, neither mutation alone determined ECG changes in the parents. Only the combined effect of the two variants resulted in a sufficient loss of sodium current recognizable at surface ECG, albeit not associated with a typical BrS clinical phenotype. Hence, our results suggest that the interactions between genetic mutations, environmental factors and phenotypic expression are increasingly complex, thus requiring collection and association of extensive genetic and clinical data.

\section{Study limitations}

The results of our study show a clear loss of current by Del4 and FS, alone and in combination, compared with WT channels. However, caution should be exercised when translating in vitro data to clinical setting. Several factors may explain the discrepancy between the strong loss of function observed in in vitro expression systems and the minimal phenotypic expression observed in mutation carriers. HEK293 cells used in the present study expressed only the alpha subunit of the sodium channel, whereas cardiac myocytes have additional proteins that were not present in this in vitro model. In addition, the voltage-clamp protocol and the perfusion temperature used in the study were far different from the physiological conditions. Decreased current resulting from the co-expression of FS+Del4 might also be due to difference in transfection efficiency or to interactions between the two plasmids. Further clinical and genetic studies with longer follow-up periods will be required to evaluate the predictive value of SCN $5 A$ mutations in asymptomatic subjects.

\section{References}

1. Brugada P, Brugada J. Right bundle branch block, persistent ST segment elevation and sudden cardiac death: a distinct clinical and electrocardiographic syndrome. A multicenter report. J Am Coll Cardiol 1992; 20:1391-6.

2. Zipes DP, Camm AJ, Borggrefe M, et al. ACC/AHA/ESC 2006 Guidelines for management of patients with ventricular arrhythmias and the prevention of sudden cardiac death: a report of the American College of Cardiology/American Heart Association Task Force and the European Society of Cardiology Committee for Practice Guidelines (writing committee to develop Guidelines for Management of Patients With Ventricular Arrhythmias and the Prevention of Sudden Cardiac Death): developed in collaboration with the European Heart Rhythm Association and the Heart Rhythm Society. Circulation 2006;114:e385-484.

3. Probst V, Veltmann C, Eckardt L, et al. Long-term prognosis of patients diagnosed with Brugada syndrome: results from the FINGER Brugada Syndrome Registry. Circulation 2010;121:635-43.

4. Probst V, Denjoy I, Meregalli PG, et al. Clinical aspects and prognosis of Brugada syndrome in children. Circulation 2007; 115:2042-8.

5. Chen Q, Kirsch GE, Zhang D, et al. Genetic basis and molecular mechanism for idiopathic ventricular fibrillation. Nature 1998;392:293-6.

6. Gellens ME, George AL Jr, Chen LQ, et al. Primary structure and functional expression of the human cardiac tetrodotoxininsensitive voltage-dependent sodium channel. Proc Natl Acad Sci USA 1992;89: 554-8.

7. Kapplinger JD, Tester DJ, Alders M, et al. An international compendium of mutations in the SCN5A-encoded cardiac sodium channel in patients referred for Brugada syndrome genetic testing. Heart Rhythm 2010;7:33-46.

8. Wilde AA, Brugada R. Phenotypical manifestations of mutations in the genes encoding subunits of the cardiac sodium channel. Circ Res 2011;108:884-97.

9. Monteforte N, Napolitano C, Priori SG. Genetics and arrhythmias: diagnostic and prognostic applications. Rev Esp Cardiol 2012;65:278-86.

10. Antzelevitch C. The Brugada syndrome: diagnostic criteria and cellular mechanisms. Eur Heart J 2001;22:356-63.

11. Herfst LJ, Rook MB, Jongsma HJ. Trafficking and functional expression of cardiac Na+ channels. J Mol Cell Cardiol 2004;36:185-93.

12. Vohra J. Diagnosis and management of Brugada syndrome. Heart Lung Circ 2011; 20:751-6.

13. Roden DM. Human genomics and its 
impact on arrhythmias. Trends Cardiovasc Med 2004;14:112-6.

14. Wilde AA, Antzelevitch C, Borggrefe M, et al. Proposed diagnostic criteria for the Brugada syndrome: consensus report. Circulation 2002;106:2514-9.

15. Vatta M, Dumaine R, Varghese G, et al. Genetic and biophysical basis of sudden unexplained nocturnal death syndrome (SUNDS), a disease allelic to Brugada syndrome. Hum Mol Genet 2002;11:337-45.

16. Shuraih M, Ai T, Vatta M, et al. A common SCN5A variant alters the responsiveness of human sodium channels to class I antiarrhythmic agents. J Cardiovasc Electrophysiol 2007;18:434-40.

17. Priori SG, Napolitano C, Gasparini M, et al. Natural history of Brugada syndrome: insights for risk stratification and management. Circulation 2002;105:1342-7.

18. Ackerman MJ, Splawski I, Makielski JC, et al. Spectrum and prevalence of cardiac sodium channel variants among black, white, Asian, and Hispanic individuals: implications for arrhythmogenic susceptibility and Brugada/long QT syndrome genetic testing. Heart Rhythm 2004;1:6007 .
19. Paparella G, Sarkozy A, Brugada P. Brugada syndrome: the prognostic dilemma and value of sincope. Minerva Med 2009;100: 307-19.

20. Takehara N, Makita N, Kawabe J, et al. A cardiac sodium channel mutation identified in Brugada syndrome associated with atrial standstill. J Intern Med 2004;255: 137-42.

21. Wang DW, Makita N, Kitabatake A, et al. Enhanced $\mathrm{Na}(+)$ channel intermediate inactivation in Brugada syndrome. Circ Res 2000;87:E37-43.

22. Valdivia CR, Tester DJ, Rok BA, et al. A trafficking defective, Brugada syndromecausing SCN5A mutation rescued by drugs. Cardiovasc Res 2004;62:53-62.

23. Baroudi G, Acharfi S, Larouche C, Chahine M. Expression and intracellular localization of an SCN5A double mutant R1232W/T1620M implicated in Brugada syndrome. Circ Res 2002;90:E11-6.

24. Yokoi H, Makita N, Sasaki K, et al. Double SCN5A mutation underlying asymptomatic Brugada syndrome. Heart Rhythm 2005;2: 285-92.

25. Bezzina CR, Rook MB, Groenewegen WA, et al. Compound heterozygosity for muta- tions (W156X and R225W) in SCN5A associated with severe cardiac conduction disturbances and degenerative changes in the conduction system. Circ Res 2003;92: 159-68.

26. Cordeiro JM, Barajas-Martinez H, Hong K, et al. Compound heterozygous mutations P336L and I1660V in the human cardiac sodium channel associated with the Brugada syndrome. Circulation 2006;114: 2026-33.

27. Barajas-Martinez HM, Hu D, Cordeiro JM, et al. Lidocaine-induced Brugada syndrome phenotype linked to a novel double mutation in the cardiac sodium channel. Circ Res 2008;103:396-404

28. Medeiros-Domingo A, Tan BH, IturraldeTorres $\mathrm{P}$, et al. Unique mixed phenotype and unexpected functional effect revealed by novel compound heterozygosity mutations involving SCN5A. Heart Rhythm 2009;6:1170-5.

29. Kanter RJ, Pfeiffer R, Hu D, et al. Brugadalike syndrome in infancy presenting with rapid ventricular tachycardia and intraventricular conduction delay. Circulation 2012;125:14-22. 